\title{
Nonextensive relativistic nuclear and subnuclear equation of state
}

\author{
A. Lavagno, P. Quarati, A. M. Scarfone \\ Dipartimento di Fisica, Politecnico di Torino, I-10129 Torino, Italy \\ Istituto Nazionale di Fisica Nucleare (INFN), Sezione di Torino e di Cagliari, Italy
}

(Received on 19 January, 2009)

\begin{abstract}
Following the basic prescriptions of the Tsallis' nonextensive relativistic thermodynamics, we investigate the relevance of nonextensive statistical effects on the relativistic nuclear and subnuclear equation of state. In this framework, we study the first order phase transition from hadronic to quark-gluon plasma phase by requiring the Gibbs conditions on the global conservation of the electric and the baryon charges. The relevance of small deviations from the standard extensive statistics is investigated in the context of intermediate and high energy heavy-ion collisions.
\end{abstract}

Keywords: Nonextensive thermostatistics, Relativistic nuclear equation of state, Hadron-quark phase transition

\section{INTRODUCTION}

The determination of the properties of nuclear matter as functions of densities, temperature and protons fraction is a fundamental task in nuclear and subnuclear physics. Several experimental observations and theoretical calculations clearly indicate that hadrons dissociate into a plasma of their elementary constituents, quarks and gluons (QGP), at density several times the nuclear matter density and/or at temperature above few hundreds $\mathrm{MeV}$, which is the critical temperature $T_{c}$ of the transition from the QGP phase to the hadronic gas phase and viceversa. Such a QGP is expected to have occurred in the early stages of the Universe and can be found in dense and hot stars, neutron stars, nucleus-nucleus high energy collisions where heavy ions are accelerated to relativistic energies [1]. After collision, a fireball is created which may realize the conditions of the QGP. The plasma then expands, cools, freezes-out into hadrons, photons, leptons that are detected and analyzed [2].

It is a rather common opinion that, because of the extreme conditions of density and temperature in ultrarelativistic heavy ion collisions, memory effects and long-range color interactions give rise to the presence of non-Markovian processes in the kinetic equation affecting the thermalization process toward equilibrium as well as the standard equilibrium distribution [3-5]. A rigorous determination of the conditions that produce a nonextensive behavior, due to memory effects and/or long-range interactions, should be based on microscopic calculations relative to the parton plasma originated during the high energy collisions. At this stage we limit ourselves to consider the problem from a qualitative point of view on the basis of the existing theoretical calculations and experimental evidences.

On the other hand, over the last years, there has been an increasing evidence that the generalized non-extensive statistical mechanics, proposed by Tsallis [6-8] and characterized by a power-law stationary particle distribution, can be considered as a basis for a theoretical framework appropriate to incorporate, at least to some extent and without going into microscopic dynamical description, long-range interactions, long-range microscopic memories and/or fractal space-time constraints. A considerable variety of physical issues show a quantitative agreement between experimental data and theoretical analysis based on Tsallis' thermostatistics. In particular, there is a growing interest in high energy physics applications of non-extensive statistics [9-14]. Several authors outline the possibility that experimental observations in relativistic heavy-ion collisions can reflect non-extensive statistical mechanics effects during the early stage of the collisions and the thermalization evolution of the system [5, 15-20]. In this context, it is relevant to observe that the statistical origin of the nonextensive statistics lies in the deformation of the Boltzmann entropy. Let us note that in literature other statistical generalizations are present, such as the $q$-deformed thermostatistics inspired to $q$-deformed quantum algebra and quantum groups with a different origin [21-24].

From the above considerations, it appears reasonable that in regime of high density and temperature both hadron and quark-gluon Equation Of State (EOS) can be sensibly affected by nonextensive statistical effects [25]. Furthermore, in this context it is very remarkable to observe that the relevance of these effects on the relativistic hadronic equation of state has also been recently investigated in Ref. [26].

The aim of this paper is to study the behavior of the nuclear equation of state at finite temperature and baryon density and to explore the existence of a hadron-quark mixed phase at a fixed value of the proton fraction $Z / A$.

\section{RELATIVISTIC NONEXTENSIVE THERMODYNAMICS}

In order to study, from a phenomenological point of view, experimental observable in relativistic heavy-ion collisions, in this Section we present the basic macroscopic thermodynamic variables and kinetic theory in the language of the nonextensive relativistic kinetic theory.

Let us start by introducing the particle four-flow in the phase space as [27]

$$
N^{\mu}(x)=\frac{1}{Z_{q}} \int \frac{d^{3} p}{p^{0}} p^{\mu} f(x, p),
$$

and the energy-momentum flow as

$$
T^{\mu v}(x)=\frac{1}{Z_{q}} \int \frac{d^{3} p}{p^{0}} p^{\mu} p^{v} f^{q}(x, p),
$$

where we have set $\hbar=c=1, x \equiv x^{\mu}=(t, \mathbf{x}), p \equiv p^{\mu}=$ $\left(p^{0}, \mathbf{p}\right), p^{0}=\sqrt{\mathbf{p}^{2}+m^{2}}$ being the relativistic energy and $f(x, p)$ the particle distribution function. In the above $Z_{q}=$ $\int d \Omega[f(x, p)]^{q}$ is the nonextensive partition function, $d \Omega$ 
stands for the corresponding phase space volume element and $q$ is the deformation parameter. The four-vector $N^{\mu}=(n, \mathbf{j})$ represents the probability density $n=n(x)$ (which is normalized to unity) and the probability flow $\mathbf{j}=\mathbf{j}(x)$. The energymomentum tensor contains the normalized $q$-mean expectation value of the energy density, as well as the energy flow, the momentum and the momentum flow per particle. Its expression follows directly from the definition of the mean $q$ expectation value in nonextensive statistics [7]; for this reason $T^{\mu \nu}$ it is given in terms of $f^{q}(x, p)$.

On the basis of the above definitions, one can show that it is possible to obtain a generalized non-linear relativistic Boltzmann equation [27]

$$
p^{\mu} \partial_{\mu}[f(x, p)]^{q}=C_{q}(x, p)
$$

where the function $C_{q}(x, p)$ implicitly defines a generalized nonextensive collision term

$$
\begin{aligned}
C_{q}(x, p)= & \frac{1}{2} \int \frac{d^{3} p_{1}}{p_{1}^{0}} \frac{d^{3} p^{\prime}}{p^{\prime}} \frac{d^{3} p^{\prime}{ }_{1}}{p^{\prime 0}}\left\{h_{q}\left[f^{\prime}, f^{\prime}{ }_{1}\right] W\left(p^{\prime}, p^{\prime}{ }_{1} \mid p, p_{1}\right)\right. \\
& \left.-h_{q}\left[f, f_{1}\right] W\left(p, p_{1} \mid p^{\prime}, p^{\prime}{ }_{1}\right)\right\}
\end{aligned}
$$

Here $W\left(p, p_{1} \mid p^{\prime}, p^{\prime}{ }_{1}\right)$ is the transition rate between a twoparticle state with initial four-momenta $p$ and $p_{1}$ and a final state with four-momenta $p^{\prime}$ and $p^{\prime}{ }_{1} ; h_{q}\left[f, f_{1}\right]$ is the $q$ correlation function relative to two particles in the same space-time position but with different four-momenta $p$ and $p_{1}$, respectively. Such a transport equation conserves the probability normalization (number of particles) and is consistent with the energy-momentum conservation laws in the framework of the normalized $q$-mean expectation value. Moreover, the collision term contains a generalized expression of the molecular chaos and for $q>0$ implies the validity of a generalized $\mathrm{H}$-theorem, if the following, nonextensive, local four-density entropy is assumed (henceforward we shall set Boltzmann constant $k_{B}$ to unity)

$$
S_{q}^{\mu}(x)=-\int \frac{d^{3} p}{p^{0}} p^{\mu} f[(x, p)]^{q}\left[\ln _{q} f(x, p)-1\right],
$$

where we have used the definition $\ln _{q} x=\left(x^{1-q}-1\right) /(1-q)$, the inverse function of the Tsallis' $q$-exponential function

$$
e_{q}(x)=[1+(1-q) x]^{1 /(1-q)}
$$

which satisfies the property $e_{q}\left(\ln _{q} x\right)=x$.

The above expression is written in a covariant form, in fact $S_{q}^{\mu}=\left(S_{q}^{0}, S_{q}^{i}\right)$, with $i=1,2,3$, and correctly transforms as a four-vector under Lorentz transformations [27], where $S_{q}^{0}$ is the relativistic Tsallis nonextensive local entropy density and $S_{q}^{i}$ is the Tsallis entropy flow. Note that for $q \rightarrow 1$, Eq.(5) reduces to the well known four-flow entropy expression [28].

At equilibrium, the solution of the above Boltzmann equation is a relativistic Tsallis-like (power law) distribution and can be written as

$$
f_{e q}(p)=\frac{1}{Z_{q}}\left[1-(1-q) \frac{p^{\mu} U_{\mu}}{T}\right]^{1 /(1-q)},
$$

where $U_{\mu}$ is the hydrodynamic four-velocity [28] and $f_{e q}$ depends only on the momentum in the absence of an external field. At this stage, $T$ is a free parameter and only in the derivation of the equation of state it will be identified with the physical temperature.

We are able now to evaluate explicitly all other thermodynamic variables and provide a complete macroscopic description of a relativistic system at the equilibrium. Considering the decomposition of the energy-momentum tensor: $T^{\mu \nu}=\varepsilon U^{\mu} U^{\nu}-P \Delta^{\mu \nu}$, where $\varepsilon$ is the energy density, $P$ the pressure and $\Delta^{\mu \nu}=g^{\mu \nu}-U^{\mu} U^{v}$, the equilibrium pressure can be calculated as

$$
P=-\frac{1}{3} T^{\mu v} \Delta_{\mu v}=-\frac{1}{3 Z_{q}} \int \frac{d^{3} p}{p^{0}} p^{\mu} p^{v} \Delta_{\mu v} f_{e q}^{q}(p) .
$$

Setting $\tau=p^{0} / T$ and $z=m / T$, the above integral can be expressed as

$$
P=\frac{4 \pi}{Z_{q}} m^{2} T^{2} K_{2}(q, z),
$$

where we have introduced the $q$-modified Bessel function of the second kind as follows

$$
K_{n}(q, z)=\frac{2^{n} n !}{(2 n) !} \frac{1}{z^{n}} \int_{z}^{\infty} d \tau\left(\tau^{2}-z^{2}\right)^{n-1 / 2}\left(e_{q}^{-\tau}\right)^{q}
$$

and $e_{q}(x)$ is the $q$-modified exponential defined in Eq.(6).

Similarly, the energy density $\varepsilon$ can be obtained from the following expression

$$
\varepsilon=T^{\mu v} U_{\mu} U_{v}=\frac{1}{Z_{q}} \int \frac{d^{3} p}{p^{0}}\left(p^{\mu} U_{\mu}\right)^{2} f_{e q}^{q}(p),
$$

and, after performing the integration, it can be cast into the compact expression:

$$
\varepsilon=\frac{4 \pi}{Z_{q}} m^{4}\left[3 \frac{K_{2}(q, z)}{z^{2}}+\frac{K_{1}(q, z)}{z}\right] .
$$

Thus the energy per particle $e=\varepsilon / n$ is

$$
e=3 T+m \frac{K_{1}(q, z)}{K_{2}(q, z)},
$$

which has the same structure of the relativistic expression obtained in the framework of the equilibrium Boltzmann-Gibbs statistics [28].

In the non-relativistic limit $(p \ll 1)$ the energy per particle reduces to the well-known expression

$$
e \simeq m+\frac{3}{2} T
$$

and no explicit $q$-dependence is left over.

Hence from the above results it appears that, in searching for the relevance of nonextensive statistical effects, both microscopic observable, such as particle distribution, correlation functions, fluctuations of thermodynamical variables, and macroscopic variables, such as energy density or pressure, can be affected by the deformation parameter $q$.

In this context, it appears relevant to observe that, in Ref. [14] nonextensive Boltzmann equation has been studied and proposed for describing the hadronization of quark matter. Moreover, starting from the above generalized relativistic 
kinetic equations, in Ref.[18] the authors have recently formulated a nonextensive hydrodynamic model for multiparticle production processes in relativistic heavy-ion collisions. These works represent an important bridge for a close connection between a microscopic nonextensive model and experimental observable.

Finally, let us remind the reader that for a system of particles in a degenerate regime the above classical distribution function (7) has to be modified by including the fermion and boson quantum statistical prescriptions. For a dilute gas of particles and/or for small deviations from the standard extensive statistics $(q \approx 1)$ the equilibrium distribution function, in the grand canonical ensemble, can be written as [29]

$$
n(k, \mu)=\frac{1}{[1+(q-1)(E(k)-\mu) / T]^{1 /(q-1)} \pm 1},
$$

where the sign + stands for fermions and - for bosons: hence all previous results can be easily extended to the case of quantum statistical mechanics.

\section{NONEXTENSIVE NUCLEAR EQUATION OF STATE}

The EOS at densities below the saturation density of nuclear matter $\rho_{0} \approx 0.16 \mathrm{fm}^{-3}$ is relatively well known due to the large amount of experimental nuclear data available. At larger densities there are many uncertainties due the lack of experimental data; the strong repulsion at short distances of nuclear force makes, in fact, the compression of nuclear matter to larger densities quite difficult. Otherwise, in relativistic heavy ion collision the baryon densities can reach values of a few times $\rho_{0}$ and the temperature can exceed the deconfinement critical temperature $T_{c} \approx 170 \mathrm{MeV}$.

As partially discussed in the Introduction, hadronic matter is expected to undergo a phase transition into a deconfined phase of quarks and gluons at large densities and/or high temperatures. However, the extraction of experimental information about the EOS of matter at large densities and temperatures at intermediate and high energy heavy-ion collisions is very complicated. Possible indirect indications of a softening of the EOS at the energies reached at AGS have been discussed several times in the literature [30, 31]. In particular, a recent analysis [32] based on a 3-fluid dynamics simulation suggests a progressive softening of the EOS tested through heavy-ion collisions at energies ranging from $2 \mathrm{~A} \mathrm{GeV}$ up to $8 \mathrm{~A} \mathrm{GeV}$. On the other hand, the information coming from experiments with heavy-ions at intermediate and high energy collisions is that, for symmetric or nearly symmetric nuclear matter, the critical density (at low temperatures) appears to be considerably larger than nuclear matter saturation density $\rho_{0}$. Concerning non-symmetric matter, general arguments based on Pauli principle suggest that the critical density decreases with $Z / A$. Therefore, the transition's critical densities are expected to sensibly depend on the isospin of the system [33]. Moreover, the analysis of observations of neutron stars, which are composed of $\beta$-stable matter for which $Z / A \leq 0.1$ (the matter constituting neutron stars is strongly isospin asymmetric, being composed of a large amount of neutrons and a small fraction of protons) can also provide hints on the structure of extremely asymmetric matter at high density. No data on the quark deconfinement transition are at the moment available for intermediate values of $Z / A$. Recently, it has been proposed by several groups to produce unstable neutron-rich beams at intermediate energies. These new experiments can open the possibility to explore in laboratory the isospin dependence of the critical densities.

The scenario we are going to explore in this last Section corresponds to the situation realized in experiments at not too high energy. In this condition, only a small fraction of strangeness can be produced and, therefore, we limit ourselves to study the deconfinement transition from nucleonic matter into up and down quark matter. In the next two subsections, we will study the two corresponding EOSs separately, on the basis of the previously reported nonextensive relativistic thermodynamic relations. The existence of the hadronquark mixed phase will be studied in the third subsection. This investigation may be helpful also in view of the future experiments planned, e.g., at the facility FAIR at GSI [34].

\subsection{Nonextensive hadronic equation of state}

The relativistic, field theoretical approach to nuclear EOS was used first by Walecka and Boguta-Bodmer in the mid1970s [35, 36]. This theory describes the interaction between nucleons through the exchange of two mesons, the scalar field $\sigma$ and the vector field $\omega$. The model of Walecka has two free parameters: the two ratios between the nucleon-meson coupling constants and the masses of the mesons. The saturation density and binding energy per nucleon (calculated at the the saturation density) of nuclear matter can be fitted exactly in the simplest version of this model but other properties of nuclear matter, as e.g. incompressibility, cannot be reproduced. To overcome these difficulties, the model has been modified introducing in the Lagrangian two terms of self-interaction for the $\sigma$ which are crucial to reproduce the empirical incompressibility of nuclear matter and the effective mass of nucleons $M^{*}$ (again calculated at the saturation density). Moreover, the introduction of an isovector meson $\rho$ allows to reproduce the correct value of the empirical symmetry energy [37, 38].

In the following, we will use a relativistic mean field selfconsistent theory of nuclear matter in which nucleons interact through the nuclear force mediated by the exchange of virtual isoscalar and isovector mesons $(\sigma, \omega, \rho)$ with a Lagrangian density [38]

$$
\begin{aligned}
\mathcal{L}= & \bar{\psi}\left[i \gamma_{\mu} \partial^{\mu}-\left(M-g_{\sigma} \sigma\right)-g_{\omega} \gamma_{\mu} \omega^{\mu}-g_{\rho} \gamma^{\mu} \vec{\tau} \cdot \vec{\rho}_{\mu}\right] \psi \\
& +\frac{1}{2}\left(\partial_{\mu} \sigma \partial^{\mu} \sigma-m_{\sigma}^{2} \sigma^{2}\right)-U(\sigma)+\frac{1}{2} m_{\omega}^{2} \omega_{\mu} \omega^{\mu} \\
& +\frac{1}{2} m_{\rho}^{2} \vec{\rho}_{\mu} \cdot \vec{\rho}^{\mu}-\frac{1}{4} F_{\mu v} F^{\mu v}-\frac{1}{4} \vec{G}_{\mu v} \vec{G}^{\mu \nu}
\end{aligned}
$$

where $M=939 \mathrm{MeV}$ is the vacuum baryon mass, $\left(\sigma, \omega_{\mu}\right)$ are the isoscalar (scalar,vector) meson fields, while $\vec{\rho}_{\mu}$ is the corresponding isovector ones. The field strength tensors for the vector mesons are given by the usual expressions $F_{\mu \nu} \equiv \partial_{\mu} \omega_{v}-\partial_{v} \omega_{\mu}, \vec{G}_{\mu v} \equiv \partial_{\mu} \vec{\rho}_{v}-\partial_{v} \vec{\rho}_{\mu}$, and the $U(\sigma)$ is a nonlinear potential of $\sigma$ meson

$$
U(\sigma)=\frac{1}{3} a \sigma^{3}+\frac{1}{4} b \sigma^{4} .
$$


This last term is usually introduced to achieve a reasonable compression modulus for equilibrium nuclear matter.

The field equations in a mean field approximation are

$$
\begin{aligned}
& \left(i \gamma_{\mu} \partial^{\mu}-\left(M-g_{\sigma} \sigma\right)-g_{\omega} \gamma^{0} \omega_{0}-g_{\rho} \gamma^{0} \tau_{3} \rho_{0}\right) \psi=0 \\
& m_{\sigma}^{2} \sigma+a \sigma^{2}+b \sigma^{3}=g_{\sigma}<\bar{\psi} \psi>=g_{\sigma} \rho_{S} \\
& m_{\omega}^{2} \omega_{0}=g_{\omega}<\bar{\psi} \gamma^{0} \psi>=g_{\omega} \rho_{B} \\
& m_{\rho}^{2} \rho_{0}=g_{\rho}<\bar{\psi} \gamma^{0} \tau_{3} \psi>=g_{\rho} \rho_{I}
\end{aligned}
$$

where $\rho_{I}=\rho_{p}-\rho_{n}$ is the isospin density, $\rho_{B}$ and $\rho_{S}$ are the baryon and the scalar densities, respectively. They are given by

$$
\begin{aligned}
& \rho_{B}=2 \sum_{i=n, p} \int \frac{\mathrm{d}^{3} k}{(2 \pi)^{3}}\left[n_{i}(k)-\bar{n}_{i}(k)\right], \\
& \rho_{S}=2 \sum_{i=n, p} \int \frac{\mathrm{d}^{3} k}{(2 \pi)^{3}} \frac{M_{i}^{\star}}{E_{i}^{\star}}\left[n_{i}^{q}(k)+\bar{n}_{i}^{q}(k)\right],
\end{aligned}
$$

where $n_{i}(k)$ and $\bar{n}_{i}(k)$ are the $q$-deformed fermion particle and antiparticle distributions given in Eq.(15); more explicitly

$$
\begin{aligned}
& n_{i}(k)=\frac{1}{\left[1+(q-1)\left(E_{i}^{\star}(k)-\mu_{i}^{\star}\right) / T\right]^{1 /(q-1)}+1}, \\
& \bar{n}_{i}(k)=\frac{1}{\left[1+(q-1)\left(E_{i}^{\star}(k)+\mu_{i}^{\star}\right) / T\right]^{1 /(q-1)}+1} .
\end{aligned}
$$

The nucleon effective energy is defined as $E_{i}^{\star}(k)=$ $\sqrt{k^{2}+M_{i}^{\star 2}}$, where $M_{i}^{\star}=M_{i}-g_{\sigma} \sigma$. The effective chemical potentials $\mu_{i}^{\star}$ are given in terms of the vector meson mean fields $\mu_{i}^{\star}=\mu_{i}-g_{\omega} \omega_{0} \mp g_{\rho} \rho_{0}$ ( - proton, + neutron), where $\mu_{i}$ are the thermodynamical chemical potentials $\mu_{i}=\partial \varepsilon / \partial \rho_{i}$. At zero temperature they reduce to the Fermi energies $E_{F i} \equiv$ $\sqrt{k_{F i}^{2}+M_{i}^{\star 2}}$ and the nonextensive statistical effects disappear. The meson fields $\left(\sigma, \omega_{0}\right.$ and $\left.\rho_{0}\right)$ are obtained as a solution of the field equations in mean field approximation and the related couplings $\left(g_{\sigma}, g_{\omega}\right.$ and $\left.g_{\rho}\right)$ are the free parameter of the model [38].

On the basis of Eqs.(2), (8) and (11), the pressure and the energy density can be written as

$$
\begin{aligned}
P & =\frac{2}{3} \sum_{i=n, p} \int \frac{\mathrm{d}^{3} k}{(2 \pi)^{3}} \frac{k^{2}}{E_{i}^{\star}(k)}\left[n_{i}^{q}(k)+\bar{n}_{i}^{q}(k)\right] \\
& -\frac{1}{2} m_{\sigma}^{2} \sigma^{2}-U(\sigma)+\frac{1}{2} m_{\omega}^{2} \omega_{0}^{2}+\frac{1}{2} m_{\rho}^{2} \rho_{0}^{2}, \\
\varepsilon & =2 \sum_{i=n, p} \int \frac{\mathrm{d}^{3} k}{(2 \pi)^{3}} E_{i}^{\star}(k)\left[n_{i}^{q}(k)+\bar{n}_{i}^{q}(k)\right] \\
& +\frac{1}{2} m_{\sigma}^{2} \sigma^{2}+U(\sigma)+\frac{1}{2} m_{\omega}^{2} \omega_{0}^{2}+\frac{1}{2} m_{\rho}^{2} \rho_{0}^{2},
\end{aligned}
$$

Note that statistical mechanics enters as an external ingredient in the functional form of the "free" particle distribution of Eq. (15). Since all the equations must be solved in a selfconsistent way, the presence of nonextensive statistical effects in the particle distribution function influences the many-body interaction in the mean field self-consistent solutions obtained for the meson fields.

In Fig. 1, we report the resulting hadronic EOS: pressure as a function of baryon chemical potential for different values of

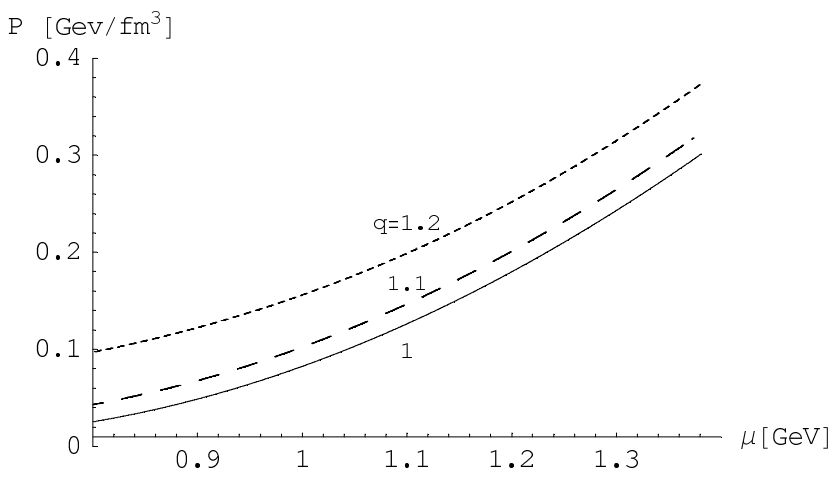

FIG. 1: Hadronic equation of state: pressure versus baryon chemical potential for different values of $q$. In the figure $T=100 \mathrm{MeV}$ and $Z / A=0.4$

$q$. Since in the previous investigations we have phenomenologically obtained values of $q$ greater than unity $[5,20]$, we will concentrate our analysis to $q>1$. The results are plotted at the temperature $T=100 \mathrm{MeV}$, at fixed value of $Z / A=0.4$ and we have used the GM2 set of parameters of Ref.[38]. The range of the considered baryon density and the chosen values of the parameters correspond to a physical situation which can be realized in the recently proposed high energy heavyion collisions experiment at GSI [39].

\subsection{Nonextensive QGP equation of state}

In high density hadronic matter, baryons are forced to stay so close one to another that they would overlap. At such densities, constituent quarks are shared by neighboring baryons and should eventually become mobile over a distance larger than the typical size of one baryon. This means that quarks become deconfined and that at large densities and/or high temperatures they are the real degrees of freedom of strongly interacting matter instead of baryons. The process of deconfinement and the EOS of quark matter can in principle be described by quantum chromodynamics. However, in energy density range reached in the core of compact stars and in relativistic heavy-ion collisions, non-perturbative effects in the complex theory of QCD are present. For these reasons, simple phenomenological models are usually adopted to describe quark matter [40-42].

In this study, due to its simplicity, we use the MIT bag model [40]. In this model, quark matter is described as a gas of free quarks with massless up and down quarks. All the non-perturbative effects are simulated by the bag constant $B$ which represents the pressure of the vacuum. Following this line, the pressure, energy density and baryon number density for a relativistic Fermi gas of quarks in the framework of nonextensive statistics (see Eqs.(1), (2), (8) and (11)) can be 
written, respectively, as

$$
\begin{aligned}
& P=\frac{\gamma_{f}}{3} \sum_{f=u, d} \int_{0}^{\infty} \frac{\mathrm{d}^{3} k}{(2 \pi)^{3}} \frac{k^{2}}{e_{f}}\left[n_{f}^{q}(k)+\bar{n}_{f}^{q}(k)\right]-B, \\
& \varepsilon=\gamma_{f} \sum_{f=u, d} \int_{0}^{\infty} \frac{\mathrm{d}^{3} k}{(2 \pi)^{3}} e_{f}\left[n_{f}^{q}(k)+\bar{n}_{f}^{q}(k)\right]+B, \\
& \rho=\frac{\gamma_{f}}{3} \sum_{f=u, d} \int_{0}^{\infty} \frac{\mathrm{d}^{3} k}{(2 \pi)^{3}}\left[n_{f}(k)-\bar{n}_{f}(k)\right],
\end{aligned}
$$

where the quark degeneracy for each flavor is $\gamma_{f}=6, e_{f}=$ $\left(k^{2}+m_{f}^{2}\right)^{1 / 2}, n_{f}(k)$ and $\bar{n}_{f}(k)$ are the $q$-deformed particle and antiparticle quark distributions

$$
\begin{aligned}
& n_{f}(k)=\frac{1}{\left[1+(q-1)\left(e_{f}(k)-\mu_{f}\right) / T\right]^{1 /(q-1)}+1}, \\
& \bar{n}_{f}(k)=\frac{1}{\left[1+(q-1)\left(e_{f}(k)+\mu_{f}\right) / T\right]^{1 /(q-1)}+1} .
\end{aligned}
$$

Similar expressions for the pressure and the energy density can be written for the gluons treating them as a massless $q$ deformed Bose gas with zero chemical potential. Explicitly, we can calculate the nonextensive pressure $P_{g}$ and density energy $\varepsilon_{g}$ for gluons as

$$
\begin{aligned}
P_{g} & =\frac{\gamma_{g}}{3} \int_{0}^{\infty} \frac{\mathrm{d}^{3} k}{(2 \pi)^{3}} \frac{k}{[1+(q-1) k / T]^{q /(q-1)}-1}, \\
\varepsilon_{g} & =3 P_{g},
\end{aligned}
$$

with the gluon degeneracy factor $\gamma_{g}=16$. Let us observe that, in the limit $q \rightarrow 1$, we recover the usual analytical expression for the pressure of gluons: $P_{g}=8 \pi^{2} / 45 T^{4}$.

Since one has to employ the fermion (boson) nonextensive distributions, the results are not analytical, even in the massless quark approximation. Hence a numerical evaluations of the integrals in Eq.s (24)-(26) and Eq.s (29)-(30) must be performed. Let us remember that a similar calculation, only for the quark-gluon phase, was also performed in Ref.[45] by studying the phase transition diagram.

As previously discussed, in this investigation we are limiting our study to the two-flavor $(f=u, d)$ massless quarks. As already remarked, this appears rather well justified for the application to heavy ion collisions at relativistic (but not ultrarelativistic) energies, the fraction of strangeness produced at these energies being small [43, 44].

In Fig. 2, we report the pressure as a function of the baryon chemical potential for massless quarks $u, d$ and gluons, for different values of $q$. As in Fig. 1, the results are plotted at the temperature $T=100 \mathrm{MeV}$ and at a fixed value of $Z / A=0.4$; the bag parameter is $B^{1 / 4}=170 \mathrm{MeV}$. In both figures 1 and 2 one can observe sizable effects in the behavior of the EOS even for small deviations from the standard statistics.

\subsection{Mixed hadron-quark phase}

In this subsection we investigate the hadron-quark phase transition at finite temperature and baryon chemical potential by means of the previous relativistic EOSs. Lattice calculations predict a critical phase transition temperature $T_{c}$ of about

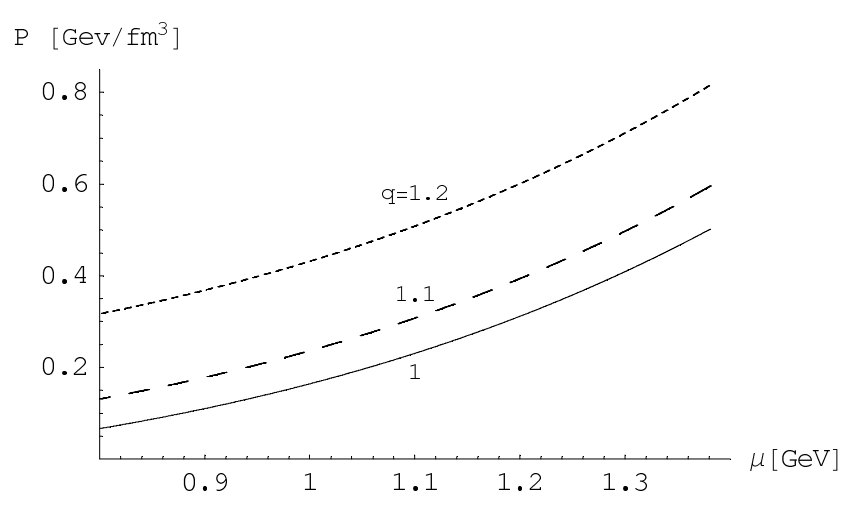

FIG. 2: The same as in Fig. 1 for the case of the quark-gluon equation of state.

$170 \mathrm{MeV}$, corresponding to a critical energy density $\varepsilon_{c} \approx 1$ $\mathrm{GeV} / \mathrm{fm}^{3}$ [1]. In a theory with only gluons and no quarks, the transition turns out to be of first order. In nature, since the $u$ and $d$ quarks have a small mass, while the strange quark has a somewhat larger mass, the phase transition is predicted to be a smooth cross over. However, since it occurs over a very narrow range of temperatures, the transition, for several practical purposes, can still be considered of first order. Indeed the lattice data with 2 or 3 dynamical flavours are not precise enough to unambigously control the difference between the two situations. Thus, by considering the deconfinement transition at finite density as a the first order one, a mixed phase can be formed, which is typically described using the two separate equations of state, one for the hadronic and one for the quark phase.

To describe the mixed phase we use the Gibbs formalism, which in Ref. [46] has been applied to systems where more than one conserved charge is present. In this contribution we are studying the formation of a mixed phase in which both baryon number and isospin charge are preserved. The main result of this formalism is that, at variance with the so-called Maxwell construction, the pressure in the mixed phase is not constant and therefore the nuclear incompressibility does not vanish. It is important to notice that from the viewpoint of Ehrenfest's definition, a phase transition with two conserved charges is considered, in the literature, not of first, but of second order [47].

The structure of the mixed phase is obtained by imposing the Gibbs conditions for chemical potentials and pressure and by requiring the global conservation of the total baryon (B) and isospin densities (I) in the hadronic phase $(\mathrm{H})$ and in the quark phase $(\mathrm{Q})$

$$
\begin{aligned}
& \mu_{B}^{(H)}=\mu_{B}^{(Q)}, \\
& \mu_{I}^{(H)}=\mu_{I}^{(Q)}, \\
& P^{(H)}\left(T, \mu_{B, I}^{(H)}\right)=P^{(Q)}\left(T, \mu_{B, I}^{(Q)}\right), \\
& \rho_{B}=(1-\chi) \rho_{B}^{H}+\chi \rho_{B}^{Q}, \\
& \rho_{I}=(1-\chi) \rho_{I}^{H}+\chi \rho_{I}^{Q},
\end{aligned}
$$

where $\chi$ is the fraction of quark matter in the mixed phase. In this way we can obtain the binodal surface which gives the 


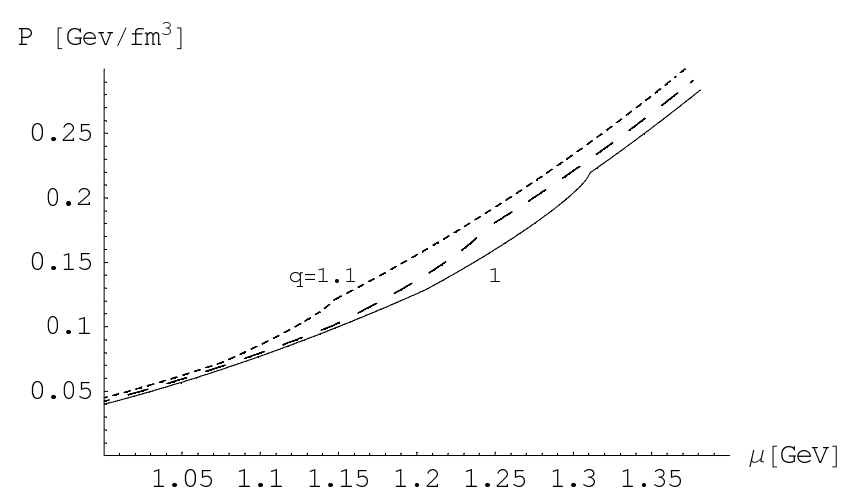

FIG. 3: Pressure versus baryon chemical potential in the mixed hadron-quark phase for different values of $q$. The long-dashed line corresponds to $q=1.05$. The temperature is fixed a $\mathrm{T}=60 \mathrm{MeV}$ and the proton fraction $Z / A=0.4$.

phase coexistence region in the $\left(T, \rho_{B}, \rho_{I}\right)$ space. For a fixed value of the conserved charge $\rho_{I}$, related to the proton fraction $Z / A \equiv\left(1+\rho_{I} / \rho_{B}\right) / 2$, we study the boundaries of the mixed phase region in the $\left(T, \rho_{B}\right)$ plane. We are particularly interested in the lower baryon density (or baryon chemical potential) border, i.e. the critical/transition density $\rho_{c r}$, in order to check the possibility of reaching such $\left(T, \rho_{c r}, \rho_{I}\right)$ conditions in a transient state during a heavy-ion collision at relativistic energies.

In Fig. 3, we report the pressure versus the baryon chemical potential, in Fig. 4, the pressure as a function of the energy density and in Fig. 5 we report the pressure versus baryon density (in units of the nuclear saturation density $\rho_{0}$ ) in the mixed hadron-quark phase for different values of $q$. For the hadronic phase we have used the so-called GM2 set of parameters [38] and in the quark phase the bag parameter is fixed to $B^{1 / 4}=170 \mathrm{MeV}$. The temperature is fixed at $T=60 \mathrm{MeV}$ and the proton fraction at $Z / A=0.4$, physical values which are estimated to be realistic for high energy heavy-ion collisions. The mixed hadron-quark phase starts at $\rho=3.75 \rho_{0}$ for $q=1$, at $\rho=3.31 \rho_{0}$ for $q=1.05$ and at $\rho=2.72 \rho_{0}$ for $q=1.1$. It is important to observe that for $q=1.1$ the second critical transition density is also reached, separating the mixed phase from the pure quark-gluon matter phase, at $\rho=4.29 \rho_{0}$ while for $q=1.05$ the second critical density is reached at $\rho=5.0 \rho_{0}$ and at $\rho=5.57 \rho_{0}$ for $q=1$.
As a concluding remark we note that nonextensive statistical effects become extremely relevant at large baryon density and energy density, as the ones which can be reached in high energy collisions experiments. This fact can be an important ingredient in the realization of a hydrodynamic model as well as to obtain a deeper microscopic connection with the experimental observable.

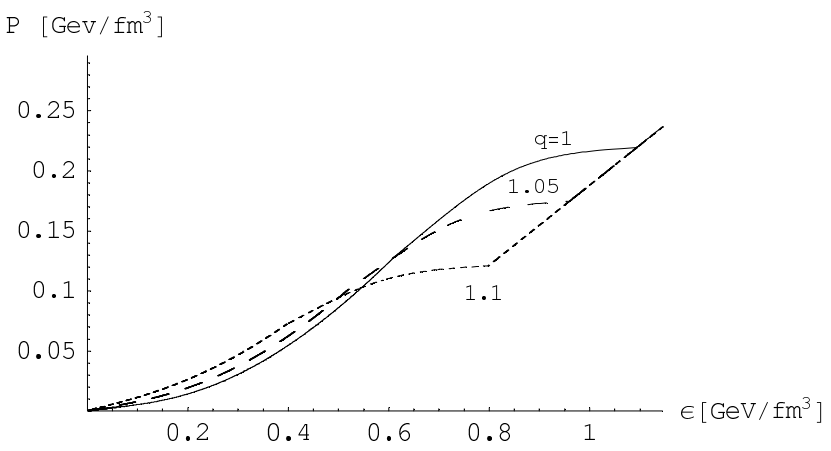

FIG. 4: Pressure versus energy density in the mixed hadron-quark phase for different values of $q$. The temperature is fixed a $T=60$ $\mathrm{MeV}$ and the proton fraction $Z / A=0.4$.

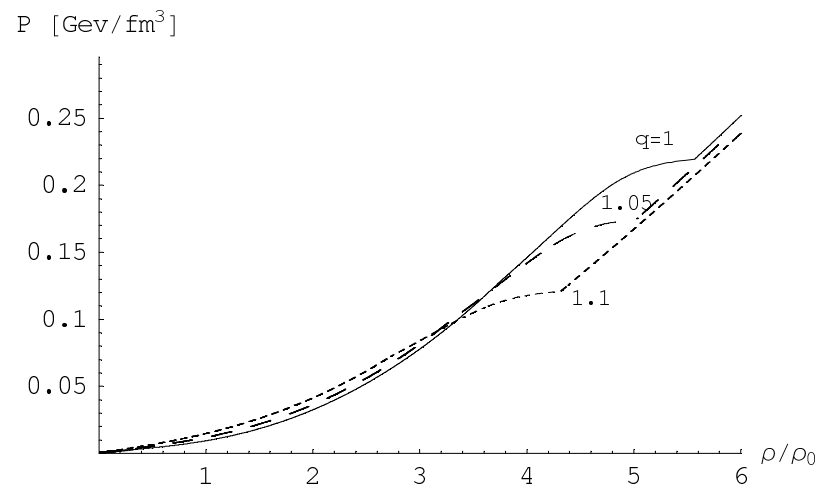

FIG. 5: Pressure versus baryon density (in units of the nuclear saturation density $\rho_{0}$ ) in the mixed hadron-quark phase for different values of $q$.
[1] R.C. Hwa and X.N. Wang, Quark Gluon Plasma 3, (World Scientific, 2004).

[2] T.S. Biró, J. Phys. G: Nucl. Part. Phys. 35, 044056 (2008).

[3] H. Heiselberg and X.N. Wang, Phys. Rev. C 53, 1892 (1996).

[4] T.S. Biró and C. Greiner, Phys. Rev. Lett. 79, 3138 (1997).

[5] W.M. Alberico, A. Lavagno and P. Quarati, Eur. Phys. J. C 12, 499 (2000); W.M. Alberico, A. Lavagno and P. Quarati, Nucl. Phys. A 680, 94c (2001).

[6] C. Tsallis, J. Stat. Phys. 52, 479 (1988).

See also http://tsallis.cat.cbpf.br/biblio.htm for a regularly updated bibliography on the subject.

[7] C. Tsallis, R.S. Mendes and A.R. Plastino, Physica A 261, 534
(1998).

[8] M. Gell-Mann and C. Tsallis (Editors), Non-extensive Entropy: Interdisciplinary Applications, (Oxford University Press, USA, 2004).

[9] G. Wilk and Z. Włodarczyk, Phys. Rev. Lett. 84, 2770 (2000); O.V. Utyuzh, G. Wilk and Z. Włodarczyk, J. Phys. G 26, L39 (2000); F.S. Navarra, O.V. Utyuzh, G. Wilk and Z. Włodarczyk, Phys. Rev. D 67, 114002 (2003).

[10] A. Lavagno and P. Quarati, Phys. Lett. B 498, 47 (2001); A. Lavagno and P. Quarati, Nucl. Phys. B [PS] 87, 209 (2000); F. Ferro, A. Lavagno and P. Quarati, Eur. Phys. A 21, 529 (2004).

[11] D.B. Walton and J. Rafelski, Phys. Rev. Lett. 84, 31 (2000). 
[12] I. Bediaga, E.M.F. Curado and J.M. de Miranda, Physica A 286, 156 (2000).

[13] C. Beck, Physica A 286, 164 (2000); Physica A 305, 209 (2002); Physica A 331, 173 (2004).

[14] T.S. Biró and G. Purcsel, Phys. Rev. Lett. 95, 162302 (2005).

[15] T.S. Biró and B. Müller, Phys. Lett. B 578, 78 (2004); T.S. Biró and A. Peshier, Phys. Lett. B 632, 247 (2006).

[16] T.S. Biró and G. Purcsel, Phys. Lett. A 372, 1174 (2008).

[17] N. Biyajima, M. Kaneyama, T. Mizoguchi and G. Wilk, Eur. Phys. J. C 40, 243 (2005); N. Biyajima et al., Eur. Phys. J. C 48, 597 (2006).

[18] T. Osada and G. Wilk, Phys. Rev. C 77, 044903 (2008).

[19] A. Lavagno, Physica A 305, 238 (2002).

[20] W.M. Alberico, P. Czerski, A. Lavagno, M. Nardi and V. Somá, Physica A 387, 467 (2008).

[21] G. Kaniadakis, A. Lavagno and P. Quarati, Phys. Lett. A 227, 227 (1997); G. Kaniadakis, A. Lavagno and P. Quarati, Nucl. Phys. B 466, 527 (1996).

[22] A. Lavagno and P. Narayana Swamy, Phys. Rev. E 61, 1218 (2000); A. Lavagno and P. Narayana Swamy, Phys. Rev. E 65, 036101 (2002).

[23] A. Lavagno, A.M. Scarfone and P. Narayana Swamy, Eur. Phys. J. C 47, 253 (2006); A. Lavagno, A.M. Scarfone and P. Narayana Swamy, J. Phys. A: Math. Theor. 40, 8635 (2007).

[24] A. Lavagno, J. Phys. A: Math. Theor. 41, 244014 (2008).

[25] A. Drago, A. Lavagno and P. Quarati, Physica A 344, 472 (2004).

[26] F.I.M. Pereira, R. Silva and J.S. Alcaniz, Phys. Rev. C 76, 015201 (2007).

[27] A. Lavagno, Phys. Lett. A 301, 13 (2002).

[28] S.R. Groot, W.A. van Leeuwen, Ch. G. van Weert, Relativistic kinetic theory, North-Holland, Amsterdam, 1980.

[29] U. Tirnakli, F. Büyükkiliç and D. Demirhan, Phys. Lett. A 245, $62(1998)$.
[30] H. Stoecker, Nucl. Phys. A 750, 121 (2005); M. Isse et al., Phys. Rev. C 72, 064908 (2005).

[31] L. Bonanno, A. Drago and A. Lavagno, Phys. Rev. Lett. 99, 242301 (2007).

[32] V. N. Russkikh and Y. B. Ivanov, Phys. Rev. C 74, 034904 (2006).

[33] M. Di Toro, A. Drago, T. Gaitanos, V. Greco and A. Lavagno, Nucl. Phys. A 775, 102 (2006); A. Drago, A. Lavagno and I. Parenti, Ap. J. 659, 1519 (2007).

[34] P. Senger, J. Phys. G 30, S1087 (2004).

[35] J.D. Walecka, Ann. of Phys. 83, 491 (1974).

[36] J. Boguta and A.R. Bodmer, Nucl. Phys. A 292, 413 (1977).

[37] B.D. Serot and J.D. Walecka, Phys. Lett. B 87, 172 (1979).

[38] N.K. Glendenning and S.A. Moszkowski, Phys. Rev. Lett. 67, 2414 (1991).

[39] GSI, Scientific Report 2007 and GSI Proposal for an International Accelerator Facility for Research with Ions and Antiprotons.

[40] A. Chodos et al., Phys. Rev. D 9, 3471 (1974).

[41] S.L. Schertler and J. Schaffner-Bielich, Phys. Rev. C 60, 025801 (1999).

[42] A. Drago and A. Lavagno, Phys. Lett. B 511, 229 (2001); A. Drago, A. Lavagno and G. Pagliara, Phys. Rev. D 69, 057505 (2004); Z. Berezhiani et al., ApJ. 586, 1250 (2003).

[43] G. Ferini, M. Colonna, T. Gaitanos and M. Di Toro, Nucl. Phys. A 762, 147 (2005); A. Drago, A. Lavagno and G. Pagliara, Phys. Rev. D 71, 103004 (2005).

[44] C. Fuchs, Prog. Part. Nucl. Phys. 56, 1 (2006).

[45] A. Teweldeberhan and H.G. Miller, R. Tegen, Int. J. Mod. Phys. E 12, 395 (2003).

[46] N. K. Glendenning, Phys. Rev. D 46, 1274 (1992).

[47] H. Müller and B. D. Serot, Phys. Rev. C 52, 2072 (1995). 